\title{
Physical Properties of Circumstellar Envelopes in Be Stars Derived from their Long-term Spectrophotometric Variations
}

\author{
A. Moujtahid \\ Observatoire de Paris-Meudon, DASGAL/UMR 8633 du CNRS, \\ F-92195 Meudon Principal Cedex, France
}

\section{J. Zorec}

Institut d'Astrophysique de Paris, CNRS, $98^{\text {bis }}$ bd. Arago, F-75014 Paris, France

\author{
A.M. Hubert \\ Observatoire de Paris-Meudon, DASGAL/UMR 8633 du CNRS, \\ F-92195 Meudon Principal Cedex, France
}

\begin{abstract}
The long-term visible spectrophotometric variations depicted by the following parameters: $V$ the apparent visual magnitude; $\Phi_{\mathrm{rb}}$ the gradient of the observed Paschen energy distribution and $D$ the total Balmer discontinuity (BD) of 49 Be stars in different spectrophotometric phases (SPh-E: spectrophotometric phase where the second component of the $\mathrm{BD}$ is in emission; SPh-A: spectrophotometric phase where the second component of the $\mathrm{BD}$ is in absorption) were translated into $\mathrm{CE}$ physical parameters. These parameters are: $R / R_{*}$ the mean extent of the CE zone that produces the visible energy distribution; $T_{\text {env }}$ its mean temperature; $\tau$ the continuum opacity at $\lambda 0.56 \mu \mathrm{m}$. The results obtained show that the triplet of parameters $\left(V, \Phi_{\mathrm{rb}}, D\right)$ do not depend on the $\left(R / R_{*}, T_{\mathrm{env}}, \tau\right)$ set of parameters in the same way if Be stars are in a SPh-E or in a SPh-A phase. Independently of the spectrophotometric phase, $T_{\text {env }}$ always decreases as a linear function of $\left(R / R_{*}\right)^{-1}$. In SPh-E it is $\left(R / R_{*}\right)^{-1} \leq 0.7$, and in SPh-A it is $\left(R / R_{*}\right)^{-1} \geq 0.7$. All SPh behaviours can be summarized by the relation $\partial T_{\text {env }} / \partial\left(R_{*} / R\right)=\partial T_{\text {env }} / \partial \tau \times \partial \tau / \partial\left(R_{*} / R\right) \geq 0$, which describes different spectrophotometric behaviours in both SPh-E and $\mathrm{SPh}-\mathrm{A}$ phases.
\end{abstract}

\section{Introduction}

The long-term spectrophotometric (SPh) changes of Be stars described by the $\left(V, \Phi_{\mathrm{rb}}, D\right)$ parameters $\left[V=\right.$ apparent magnitude; $\Phi_{\mathrm{rb}}=$ gradient of the Paschen continuum spectrum; $D=$ total Balmer discontinuity] presented in Moujtahid et al. $(1998,1999)$, mirror the variation of parameters which characterize the physical and geometrical structure of their CE. It is then important to translate 
the $\left(V, \Phi_{\mathrm{rb}}, D\right)$ triplet into relevant physical parameters and to study the possible relations among them which underly the observed $\mathrm{SPh}$ variations.

\section{Translation into physical parameters}

Using the analytical expression for the radiation flux in the continuum spectrum emitted by a star-CE system given in another contribution of this issue by Moujtahid et al., the triplet $\left(V, \Phi_{\mathrm{rb}}, D\right)$ was written as a function of: $R / R_{*}$ $=$ mean extent of the region in the CE responsible for the SPh changes; $T_{\text {env }}=$ mean temperature of the $\mathrm{CE} ; \tau_{\mathrm{e}}=$ electron scattering opacity and $\tau^{\mathrm{V}}=\tau(\mathrm{bf}+\mathrm{ff})$ true absorption opacity (boud-free+free-free) at $\lambda=0.56 \mu \mathrm{m}$ (total opacity at $\left.\lambda_{\mathrm{V}}: \tau_{\mathrm{V}}=\tau_{\mathrm{e}}+\tau^{\mathrm{V}}\right) ; \gamma=$ exponent characterizing the density distribution in the $\mathrm{CE}$. Hence, we obtained the system of equations (1), where the left hand side is supplied by the observations and the right hand is their translation into model parameters of the $\mathrm{CE}$ :

$$
\begin{aligned}
V & =V\left(R, T_{\text {env }}, \tau_{\mathrm{e}}, \tau^{\mathrm{V}}, \gamma\right) \\
\Phi_{\mathrm{rb}} & =\Phi_{\mathrm{rb}}\left(R, T_{\mathrm{env}}, \tau_{\mathrm{e}}, \tau^{\mathrm{V}}, \gamma\right) \\
D & =D\left(R, T_{\mathrm{env}}, \tau_{\mathrm{e}}, \tau^{\mathrm{V}}, \gamma\right)
\end{aligned}
$$

Considering that in most CE of Be stars $\tau_{\mathrm{e}} \gg \tau^{\mathrm{V}}$ and that $\tau^{\mathrm{V}}=f(\gamma) \tau_{\mathrm{e}}^{2}$, we can use the opacity $\tau_{\mathbf{e}}$ as the main density indicator in the CE. As the parameter $\gamma$ enters into the determination of $\tau^{V}$ opacity, whose value is low, the value of $\gamma$ is not relevant for solution of the system (1). Thus, for $\gamma$ we adopted a mean value which is slightly different for $\mathrm{SPh}$ phases where the second Balmer discontinuity is in emission ( $\mathrm{SPh}-\mathrm{E}$ phases) than for phases where the second Balmer discontinuity is in absorption (SPh-A phases).

\section{Results}

The long-term variation of the triplet $\left(V, \Phi_{\mathrm{rb}}, D\right)$ reported in Moujtahid et al. (1998) for all Be stars studied there, and which are in different SPh phases, were translated into the triplet $\left(R, T_{\text {env }}, \tau_{\mathrm{e}}\right)$ of $\mathrm{CE}$ model parameters using the system of equations (1). Fig. 1(a) shows the results for $\gamma$ Cas (HD 5394), which represents a SPh-E phase and Fig. 1(b) those for HD 83953 whose spectrophotometric variations are typical of a SPh-A phase. These figures give $T_{\text {env }}$ as functions of $R_{*} / R$, the "inverse" mean extent of the regions producing their $\mathrm{SPh}$ variations, and of $\tau_{\mathrm{e}}$, the electron scattering optical depth of these regions. The relation between $\tau_{\mathrm{e}}$ and $R_{*} / R$ is also shown. The extreme values of these parameters are indicated on the abscissas.

The most outstanding conclusions that can be drawn from the whole set of results similar to those shown in Fig. 1(a,b) are:

1. The mean radius $R / R_{*}$ of $\mathrm{CE}$ regions responsible for the observed longterm $\mathrm{SPh}$ changes are larger in $\mathrm{SPh}-\mathrm{E}$ phases than in $\mathrm{SPh}-\mathrm{A}$ phases. On average we have:

$$
\begin{aligned}
& <R / R_{*}>_{\mathrm{SPh}-\mathrm{E}} \gtrsim 1.4 \\
& <R / R_{*}>_{\mathrm{SPh}-\mathrm{A}} \lesssim 1.4
\end{aligned}
$$



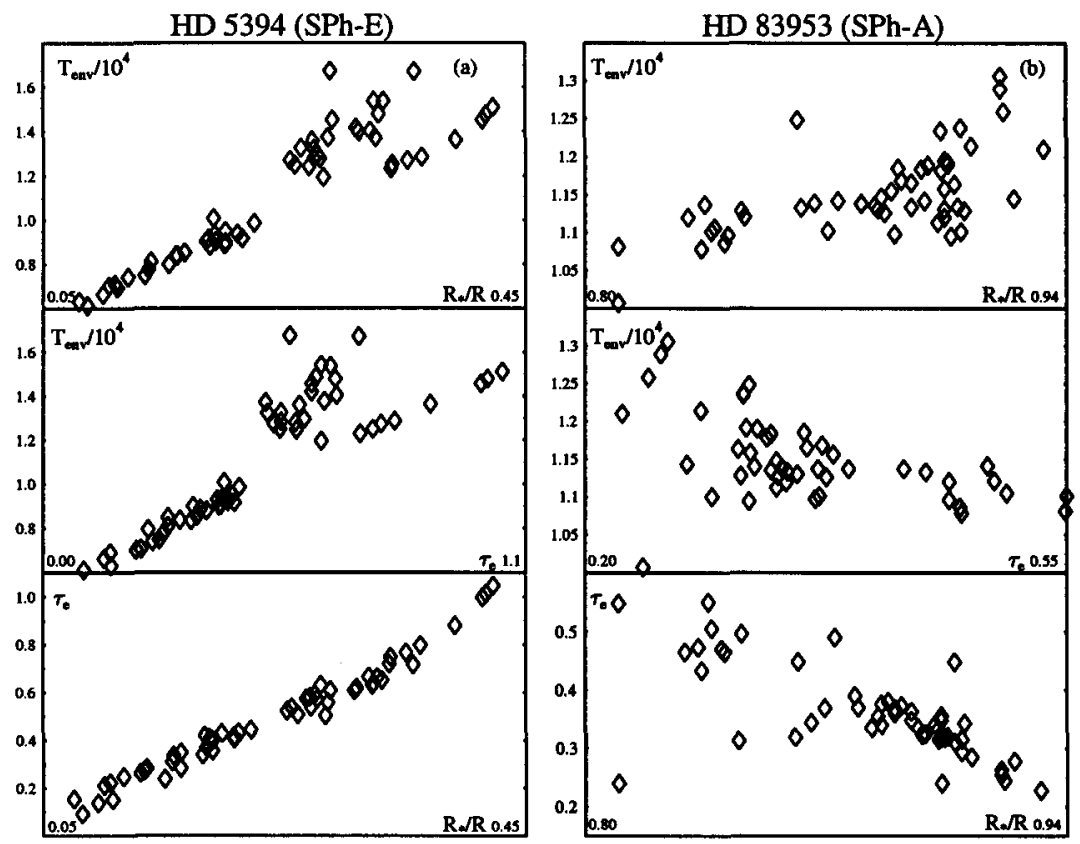

Figure 1. Relations among the CE parameters $\left(T_{\text {env }}, R_{*} / R, \tau_{\mathrm{e}}\right)$ implied in SPh variations. (a): HD 5394 (SPh-E); (b) HD 83953 (SPh-A).

2. The amplitudes of the variation of the size of CE are higher in SPh-E than in $\mathrm{SPh}-\mathrm{A}$ phases. On average:

$$
\begin{aligned}
& <\Delta R / R_{*}>_{\mathrm{SPh}-\mathrm{E}} \simeq 0.9 \\
& <\Delta R / R_{*}>_{\mathrm{SPh}-\mathrm{A}} \simeq 0.5
\end{aligned}
$$

3. The temperature ratios $T_{\text {env }} / T_{\text {eff }}$ are slightly higher in SPh-A phases than in SPh-E phases. Nevertheless, as for our set of stars it is $\overline{T_{\text {eff }}^{\text {SPh-E }}} \sim$ $1.3 \times \bar{T}_{\text {eff }}^{\mathrm{SPh}-\mathrm{A}}$ we finally have $\bar{T}_{\mathrm{env}}^{\mathrm{SPh}-\mathrm{E}} \gtrsim T_{\mathrm{env}}^{\mathrm{SPh}-\mathrm{A}}$.

4. The electron scattering opacities $\tau_{\mathrm{e}}$ are slightly higher in SPh-E phases than in SPh-A. However, the total opacities $\tau \mathrm{V}=\tau_{\mathrm{e}}+\tau^{\mathrm{V}}$ are on average about the same in both $\mathrm{SPh}$ phases.

We can also see that in SPh-E phases:

- The $V$ magnitude is the most sensitive $\mathrm{SPh}$ parameter to changes of the mean extent of the CE. It also sometimes depends on opacity, but it is almost insensitive to temperature changes.

- The gradient $\Phi_{\mathrm{rb}}$ depends almost entirely on the opacity of the CE. Sometimes it can be somewhat sensitive to the temperature, but it is insensitive to the extent changes. 
- The total Balmer discontinuity is sensitive to changes of the mean temperature $T_{\text {env }}$. It is almost insensitive to opacity, and it does not reflect any change of the extent of the $\mathrm{CE}$.

Specifically for SPh-A phases we have:

- the variation of the $V$ magnitude is generally small in SPh-A phases. Sometimes there is some variation of $V$ at Balmer discontinuity differences $\Delta D=D-D_{*} \leq 0.1$. In these cases $V$ is sensitive to the variation of $\tau_{\mathrm{e}}$ and only slightly to the CE extent.

- in SPh-A the variation of $\Phi_{\mathrm{rb}}$ is very small. When there is some variation of $\Phi_{\mathrm{rb}}$ it seems to be due to opacity variations.

- the variation of $D$ is high in SPh-A phases. This variation is correlated with $T_{\text {env }}$ and with opacity.

Concerning the correlation of $\mathrm{CE}$ model parameters among themselves, we can note:

1. whatever the $\mathrm{SPh}$ phase, there is a linear correlation between $T_{\text {env }}$ and $R_{*} / R$, so that $\partial T_{\mathrm{env}} / \partial\left(R_{*} / R\right) \geq 0$. We can still distinguish two cases which depend on the relation between $T_{\text {env }}$ and $\tau$, and that of opacity with $R_{*} / R$. Let us write:

$$
\frac{\partial T_{\mathrm{env}}}{\partial\left(R_{*} / R\right)}=\frac{\partial T_{\mathrm{env}}}{\partial \tau} \times \frac{\partial \tau}{\partial\left(R_{*} / R\right)} \geq 0
$$

Both derivatives in the right hand must be simultaneously positive or negative. Cases with positive derivatives are seen in HD 5394, HD 24534, HD 58978, HD 148184. Negative derivatives are seen in HD 83953 and HD 142983.

2. we also see that negative gradients occur most frequently when $R_{*} / R \leq 0.7$ and positive ones when $R_{*} / R \geq 0.7$.

3. the condition $R_{*} / R \geq 0.7$ seems to be a rule for the appearance of SPh-A phases.

\section{Conclusion}

Values of physical parameters characterizing the CE in Be stars, as well as the relations among them implied by their $\mathrm{SPh}$ variations, were reported in this paper. As these relations differ according to the particular $\mathrm{SPh}$ phase, they may be relevant for understanding of the structure of the CE near the central stars and perhaps also to their formation.

\section{References}

Moujtahid, A., Zorec, J., Hubert, A.M. 1998, A\&AS 129, 289

Moujtahid, A., Zorec, J., Hubert, A.M. 1999, A\&A 349, 151 